\title{
Computational Analysis of Shrouded Wind Turbine Configurations Using a 3-Dimensional RANS Solver
}

\author{
Aniket C. Aranake, Vinod K. Lakshminarayan and Karthik Duraisamy \\ Department of Aeronautics and Astronautics, Stanford University, Stanford, CA 94035, U.S.A
}

\begin{abstract}
The use of a shroud is known to improve performance. In this work, the flow physics and performance of shrouded turbines is assessed by solving the Reynolds Averaged Navier-Stokes equations supplemented with a transition model. Shroud geometries are evaluated for their augmentation of mass flow through the turbine. Initial assessments are performed using axisymmetric calculations of annular wings with high-lift airfoils as cross sections. The mass flow amplification factor is defined as a performance parameter and is found to increase nearly linearly with radial lift force. From a selection of considered airfoils, the Selig S1223 high-lift airfoil is found to best promote mass flow rate. Full three-dimensional simulations of shrouded wind turbines are performed for selected shroud geometries. The results are compared to open turbine solutions. Augmentation ratios of up to 1.9 are achieved. Peak augmentation occurs at the highest wind speed for which the flow over the blade stays attached. Flow fields are examined in detail and the following aspects are investigated: regions with flow separation, the development of velocity profiles, and the interaction between the turbine wake and shroud boundary layer. The sensitivity of the solutions to rotation rate is examined.
\end{abstract}

\author{
Nomenclature \\ $\dot{m} \quad$ Mass flow rate \\ $\omega \quad$ Turbine angular velocity \\ $c_{d} \quad$ Sectional drag coefficient \\ $c_{f} \quad$ Skin friction coefficient \\ $C_{P} \quad$ Turbine power coefficient, $P / \frac{1}{2} \rho \pi R^{2} U_{\infty}^{3}$ \\ $C_{p} \quad$ Pressure coefficient \\ $C_{T} \quad$ Turbine thrust coefficient, $T / \frac{1}{2} \rho \pi R^{2} U_{\infty}^{2}$ \\ $M_{r} \quad$ Mass flow amplification factor, $\dot{m} / \rho U_{\infty} \pi R_{s}^{2}$ \\ $p \quad$ Local field pressure \\ $p_{\infty} \quad$ Freestream pressure \\ $r_{a} \quad$ Augmentation ratio, $C_{P} / 0.593$ \\ $R_{s} \quad$ Shroud radius at turbine plane \\ T Turbine thrust \\ $u_{z} \quad$ Axial component of velocity \\ $V_{\text {tip }} \quad$ Turbine tip velocity, $\omega R$ \\ $x_{\text {trans }}$ Chordwise transition location
}




\section{Introduction}

The primary obstacle presently confronting the widespread adoption of wind energy is cost. Improvements in wind turbine efficiency would lower the cost of wind power and thereby advance its suitability for investments in the energy market. At present, the most established machine for harvesting wind energy is the horizontal axis wind turbine (HAWT). As stated by the well-known Betz limit, the efficiency of conventional HAWTs is bounded above by $59.3 \%$. This limit is based on an assumption that the freestream wind is not disturbed by any external force prior to its interaction with the turbine. It has long been established, however, that altering the streamtube incident on the turbine rotor via some external mechanism can produce efficiencies in excess of the Betz limit. A shrouded wind turbine, often referred to as a diffuser augmented wind turbine (DAWT) in previous literature, makes use of such a mechanism. In a shrouded turbine, the turbine is encased in a shroud which accelerates the incoming wind, significantly increasing the mass and power available to the turbine. According to a generalized version of the classic Betz limit proposed by Jamieson [1], the theoretical limit for power extraction for a wind turbine with augmentation can be as high as $88.89 \%$ of the energy available in the streamtube incident on the rotor. (Note that this is not a limit of efficiency by the standard definition, as the upstream area of the streamtube in question will in general differ from the area of the rotor.)

In addition to the capability of exceeding the Betz limit, shrouded turbines also offer other potential advantages. Every wind turbine is limited by its so-called 'cut-in speed', a wind speed below which the blades do not turn. High cut-in speeds significantly hinder the ability of a wind operation to confidently generate power at times of high demand, a problem exacerbated by the inherent intermittency of wind. Recent progress in materials technology have enabled the construction of massively large blades, which alleviates the challenges due to cut-in speeds for new large wind farms. However, this solution is not ideal for all situations. For instance, in urban or remote settings, a smaller and more portable solution is desirable. In such cases, by accelerating the wind before it reaches the turbine, a shrouded turbine achieves a lower cut-in speed than a conventional open rotor and can operate productively for a longer portion of its lifetime.

Few researchers in the past have examined the benefits and economics of placing a diffuser around a wind turbine. A survey including a comprehensive history of shrouded turbines has been recently published by van Bussel [2]. The idea of a shrouded turbine was first proposed by Lilley and Rainbird [3]. Experimental investigations were performed in the 1980s by Gilbert and Foreman [4] and by Igra [5]. Although these experiments did demonstrate that power extraction beyond the classic Betz limit is possible, the shrouded turbine was determined not to be profitable compared to traditional wind turbines and was not pursued extensively.

Recently however, an increase in number of publications on the topic and attempts to commercialize the idea indicate a renewed interest in shrouded turbines. Researchers have come to an agreement that there is significant potential for improvements in this concept and understanding the details of flow physics is one of keys to achieving this. An investigation by Hansen [6] demonstrates using both low-fidelity momentum theory and CFD that the power augmentation of a shrouded turbine is proportional to the increase in mass flow rate through the turbine blades. Throughout the past decade, the research group of Ohya $[7,8,9,10]$ have performed extensive experimental and computational work on this topic which has led to the development of a high performance so-called "flanged diffuser".

Due to the high cost associated with experiments, computational studies are important for an in-depth understanding of the flow physics of shrouded turbines. However, to the authors knowledge, all the previous work employ modeling simplifications that affect the accuracy of flowfield predictions. Early work [5, 4] attempted to model the behavior of a shrouded wind turbine as an internal flow problem, in which a turbine was placed inside a duct with an imposed pressure condition at the duct outlet. More recently, however, it has been determined $[11,1]$ that this assumption is inaccurate, and a more appropriate boundary condition is one which is imposed far downstream of the entire system, i.e., the shrouded turbine must be treated as an external flow problem.

In some of the recent computational studies of shrouded turbines [6, 7], an actuator disk assumption is used to model the turbine blades. In such a model, the rotor is replaced by an infinitely thin disk across which a pressure drop is explicitly imposed. This simplification offers a substantial cost saving over a full three-dimensional solution, making it a useful tool during the preliminary design stage. It does not, however, capture finer and potentionally profound aspects of the flow physics near the blade and in the wake of a shrouded turbine. In addition, the actuator disk model cannot predict the effect of the turbine wake on flow 
separation along the walls of the shroud. This effect, the "swirl mixing mechanism" mentioned in previous literature [4], has potentially significant consequences on the performance of a shrouded turbine.

A fully Reynolds-Averaged-Navier-Stokes (RANS)-based CFD simulation can overcome the above mentioned modeling issues. The current work uses a compressible RANS solver to simulate shrouded wind turbines. The solver has been validated previously by Baeder [12], Duraisamy [13, 14] and Lakshminarayan $[15,16]$ for a variety of flows including single, coaxial, and shrouded rotors, primarily in the context of helicopters. While this legacy admits considerable confidence in the accuracy of the solver for rotating flows, the simulation of wind turbines presents an additional complication. This is due to free transition from laminar to turbulent flow, which occurs on wind turbine blades during typical operating conditions and is found to significantly impact both pressure and viscous forces. Recently, authors of the present work have performed a detailed validation of $3 \mathrm{D}$ simulations of an open wind turbine [17] using the $\gamma-\overline{R e_{\theta t}}-S A$ transition model [18], which is demonstrated to accurately predict transitional flows in two and three dimensions. A preliminary investigation of shrouded turbines has also been performed by present authors [19]. The primary objective of the present work is to employ this previously validated methodology to examine the performance and flowfield of shrouded wind turbines.

The remainder of this paper is organized as follows. Section 2 outlines the methodology employed by the flow solver OVERTURNS which is used throughout this work. In Section 3, a summary of the validation is presented. In Section 4, results are presented for a baseline shroud generated from a NACA0006 airfoil. In Section 5, axisymmetric computations are performed to investigate a number of different shroud geometries. Based on this analysis, the high-lift S1223 airfoil shape is selected for detailed investigation. In Section 6, a shroud generated from a S1223 airfoil section is examined in detail. The behavior of the shrouded turbine with varying tip speed ratio is investigated. Section 7 summarizes the key conclusions and proposes future work.

\section{Methodology}

In this work, computations are performed using the overset structured mesh solver OVERTURNS [15]. This code solves the compressible RANS equations using a preconditioned dual-time scheme in the diagonalized approximate factorization framework, described by Buelow et al. [20] and Pandya et al. [21]. In this framework, time is advanced using the implicit approximate factorization method developed by Pulliam and Chaussee [22]. Low Mach preconditioning, based on that developed by Turkel [23], is used to improve both convergence properties and the accuracy of the spatial discretization. Inviscid terms are computed using a third order MUSCL scheme using Koren's limiter with Roe's flux difference splitting, and the viscous terms are computed using second order central differencing. For RANS closure, the Spalart-Allmaras [24] turbulence model is employed. To capture the effects of flow transition, the $\gamma-\overline{R E_{\theta t}}-S A$ [18] model is used. The governing equations are solved in the rotating frame, with no-slip conditions accounting for wall velocities applied on the surfaces of both the shroud and turbine blade. An implicit hole-cutting technique developed by Lee [25] and improved by Lakshminarayan [15] is used to determine the connectivity information between various overlapping meshes.

\section{Assessment of Transition Model}

In this section, the performance of a newly implemented transition model is carefully evaluated. First, a performance prediction validation is performed on a two-dimensional flow over the S809 airfoil. Subsequently, full three-dimensional calculations of the flow past the NREL Phase VI turbine are performed, and the results are compared to the experimental measurements including performance and surface pressure data.

\subsection{S809 Airfoil}

The S809 airfoil, employed by the NREL Phase VI wind turbine [26], is one of a family of airfoils deliberately designed to contain a so-called "transition-ramp," a short region of gentle pressure recovery along the upper surface which induces smooth transition from laminar to turbulent flow [27]. Computations are performed using structured C-meshes with dimensions $527 \times 101$ in the wrap-around and normal directions respectively. The airfoil and mesh are shown in Figure 1. To match experimental conditions, simulations 


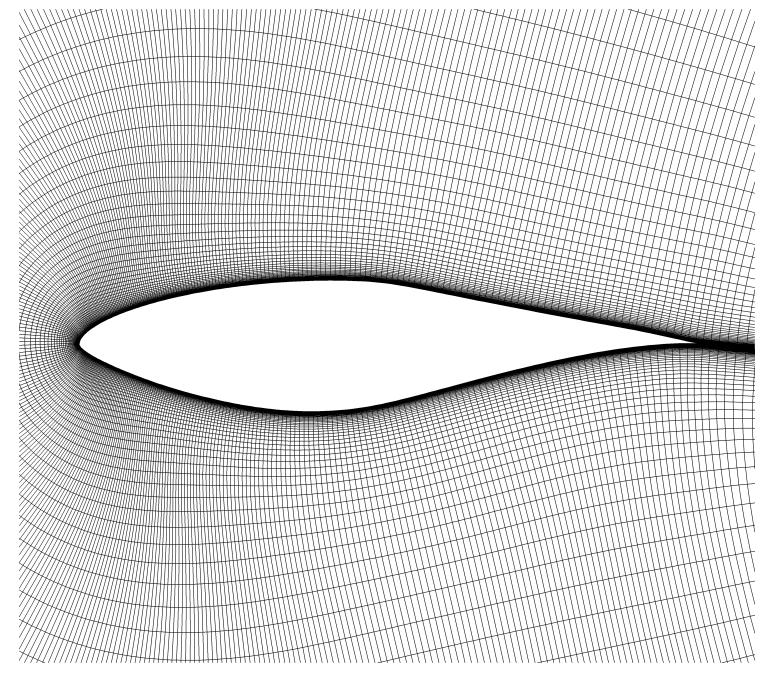

(a) S809 airfoil and near-body mesh

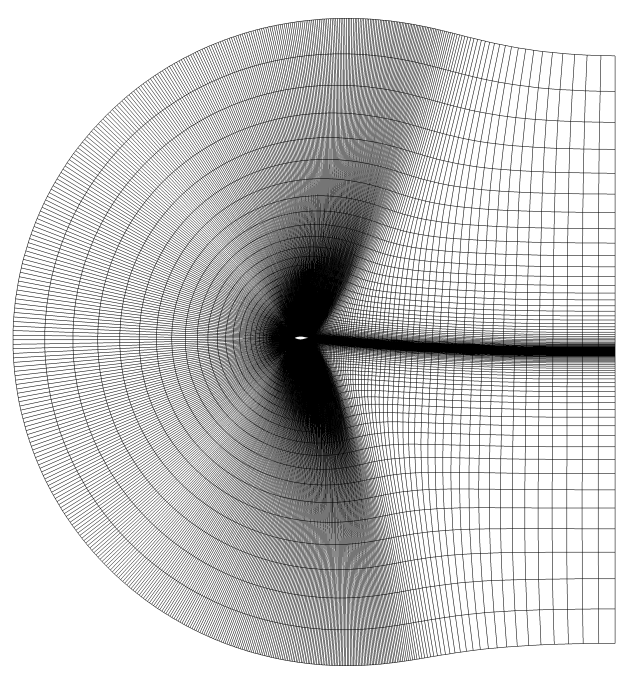

(b) Full mesh for S809 calculations

Figure 1: Computational mesh $(527 \times 101)$ for the S809 wind turbine airfoil.

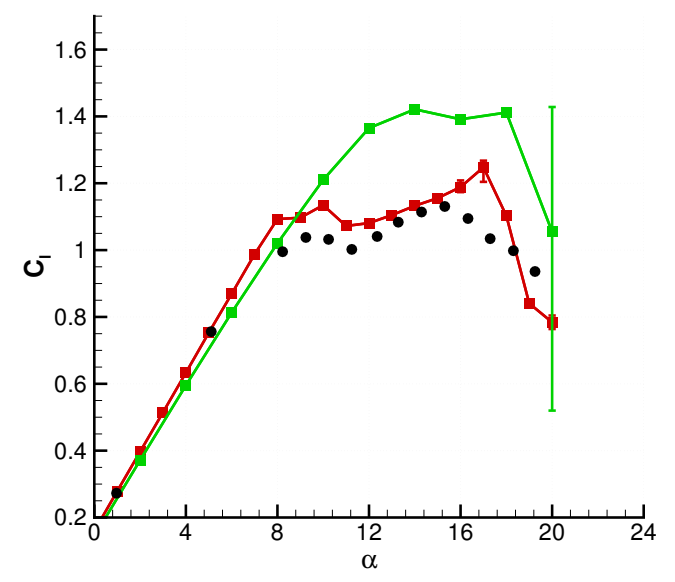

(a)

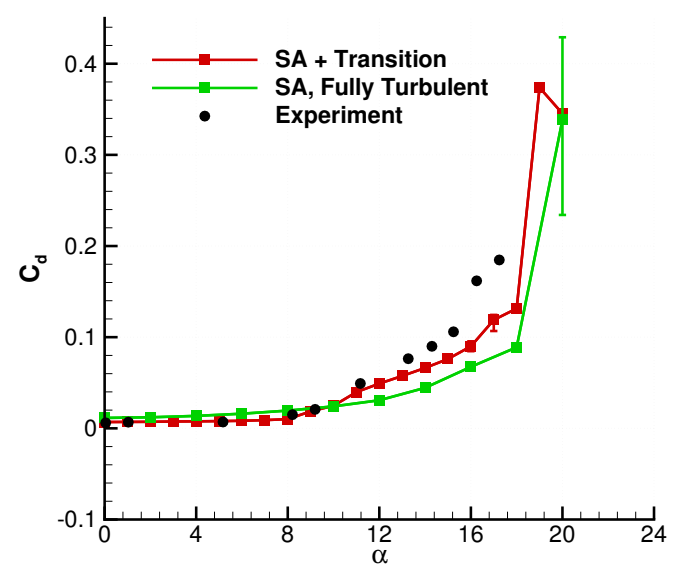

(b)

Figure 2: Lift and drag coefficients vs angle of attack for S809 airfoil. 


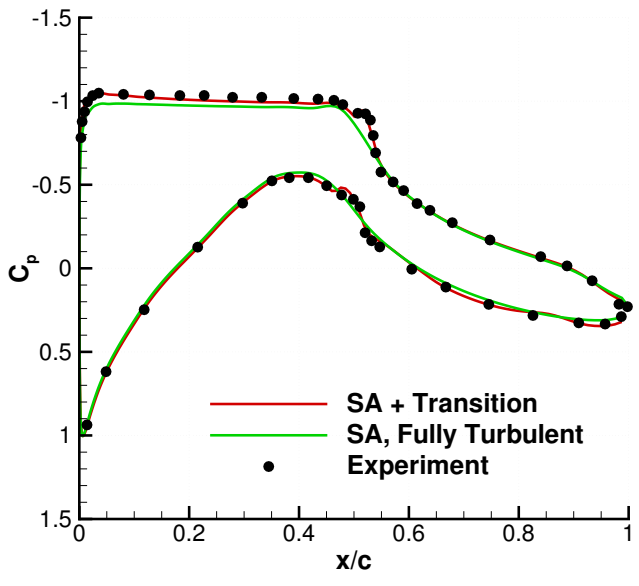

(a) $\alpha=4^{\circ}$

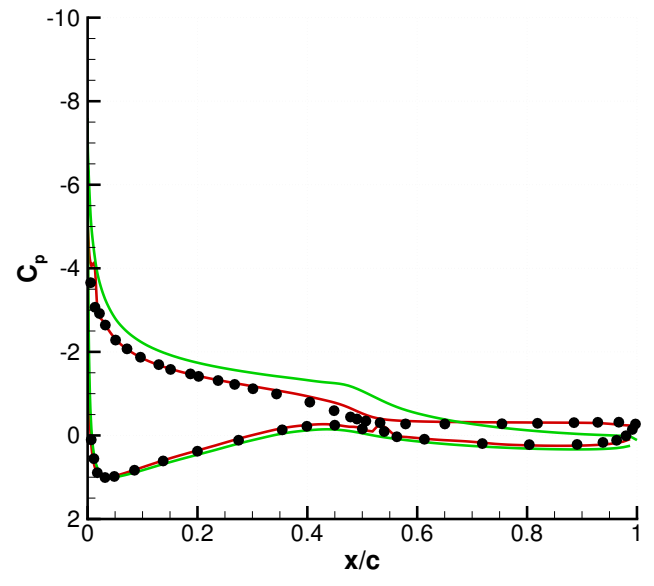

(b) $\alpha=12^{\circ}$

Figure 3: Pressure coefficient distribution for S809 airfoil.

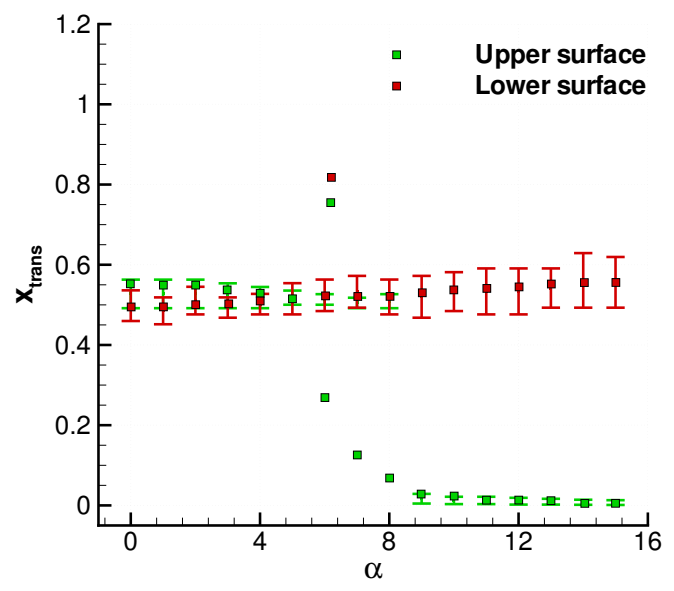

Figure 4: Transition locations for S809 airfoil. The square symbols in this plot are experimental data points. Error bars show the size of the separation bubble in the CFD solution. 
are run at a Reynolds number of $2 \times 10^{6}$, and a low Mach number of 0.1 is selected to avoid effects due to compressibility.

The predicted lift and drag coefficients for a sweep of angles of attack are shown in Figure 2. Results are plotted both with the transition model and without, in which case the flow was assumed to be fully turbulent. The $\gamma-\overline{R e_{\theta t}}-S A$ model significantly improves the prediction of both quantities, particularly in the post-stall region. The use of the transition model also improves the prediction of the pressure coefficient significantly as shown in Figure 3. At $\alpha=4^{\circ}$, shown in Figure 3(a), the prominent dip in pressure on the upper surface which indicates transition is well-captured by the transition model. Furthermore, at $\alpha=12^{\circ}$, shown in Figure 3(b), when the airfoil is partially stalled, the prediction of the entire pressure distribution is substantially improved by the inclusion of the transition model.

The location of the transition point at each angle of attack is plotted against experimental data in Figure 4. Transition is triggered by laminar separation bubbles, which span several grid points in the CFD solutions. Error bars are used to represent the location and width of these bubbles. The transition locations are captured well except in the range between $\alpha=6^{\circ}$ and $\alpha=8^{\circ}$ on the upper surface of the airfoil. The calculations predict that the separation bubble on the upper surface jumps to the leading edge of the airfoil at $\alpha=9^{\circ}$. In reality, the transition location is highly sensitive to small disturbances in the middle range of angle of attack, and even the experimental data is not single-valued in this region. Nonetheless, the transition model significantly improves the prediction of quantities from two-dimensional S809 flowfields overall.

\subsection{NREL Phase VI Wind Turbine}

For three-dimensional validation, the NREL Phase VI serves as a valuable test case, as comprehensive performance and surface pressure data is available for this configuration from the Unsteady Aerodynamics Experiment (UAE) [26]. This experiment was conducted at a large scale wind tunnel $(24.4 \mathrm{~m} \times 36.6 \mathrm{~m})$ located at NASA Ames. The tunnel maintains a freestream turbulence intensity of below $0.5 \%$. The turbine is a $2-$ bladed configuration with a radius of $R=5.029 \mathrm{~m}$. The tip Mach number is $M_{\text {tip }}=.1135$ and the rotational velocity is $\omega=72 \mathrm{rpm}$. As previously mentioned, the S809 airfoil is used for the blade cross sections. Measurements of performance and surface pressure are taken for wind speeds ranging from $U_{\infty}=3 \mathrm{~m} / \mathrm{s}$ to $25 \mathrm{~m} / \mathrm{s}$.

In this work, the turbine blade is represented by a $\mathrm{C}-\mathrm{O}$ mesh with dimensions $257 \times 51 \times 51$ in the wrap-around, spanwise, and normal directions respectively. A view of the mesh near the blade tip is presented in Figure 5(a). This mesh is embedded in a background mesh with dimensions $201 \times 133 \times 164$ in the azimuthal, radial, and axial directions respectively. The background mesh is semi-cylindrical, and periodic boundary conditions are applied on lateral faces to simulate a full two-bladed rotor. The fluid domain extends $2 R$ upstream and $6.8 R$ downstream and $3.4 R$ laterally. The azimuthal grid spacing stretches to give fine resolution in a $15^{\circ}$ patch in the vicinity of the turbine blade. Characteristic boundary conditions are used for the inflow, outflow, and farfield faces. A wall boundary condition imposes no-slip on the blade in the rotating frame. The singularity at the axis is avoided by beginning the radial point distribution at a small value of $r=0.03$. An extrapolation boundary condition is applied at this location. The background mesh and boundary conditions are depicted in Figure 5(b). To evaluate grid convergence, a finer mesh with dimensions $257 \times 201 \times 101$ for the blade and $180 \times 264 \times 228$ for the background mesh (with uniform azimuthal spacing) were run for one windspeed, and the integrated loads and pressure coefficients were all found to differ by less than $3 \%$. Since the mesh size is already very high, we did not perform more extensive refinement studies. However, all of these meshes were generated using prior experience in dealing with a number of highly similar problems with the same code over the past 15 years $[12,13,14,15,16]$.

Figure 6 (a) plots the computed torque of the open turbine at various wind speeds. These computations were performed by first attempting a steady-state solution, which in general did not converge, and then using the obtained result to restart a time-accurate solution. Error bars show the min and max values attained. For comparison, computational results from Potsdam et al. [28] are also included. At low speeds where the flow remains attached, computational results agree quite well with measured data. At these speeds, transition plays a small role in the behavior of the flow, and the transition model has little effect on the integrated results. A visualization of the wake from one such result is shown in Figure 6(b), which plots isocontours of q-criterion [29]. The helical wake vortex persists for several turns with minimal numerical diffusion. As the free stream velocity increases, transition plays a more significant role in the flow. Pressure distributions at two spanwise stations are shown in Figures 6(c) and 6(d) for a wind speed of $U_{\infty}=10 \mathrm{~m} / \mathrm{s}$. The transition 


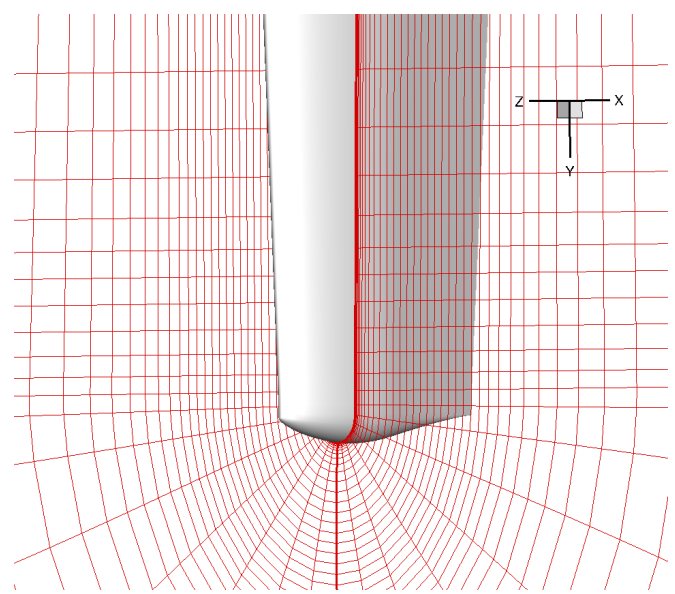

(a) Blade grid $(257 \times 51 \times 51)$

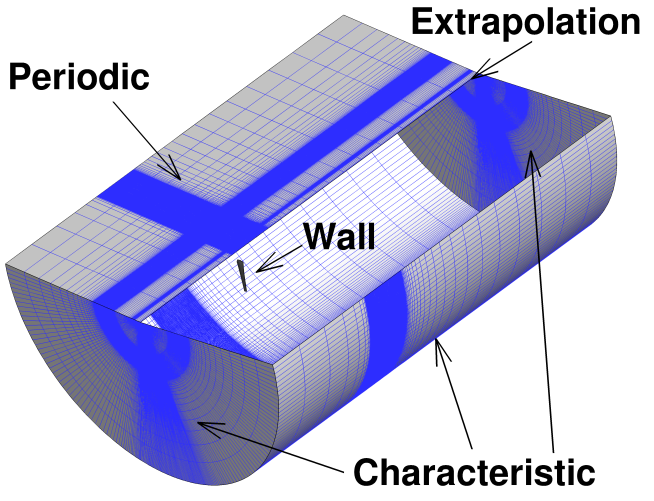

(b) Background grid $(201 \times 133 \times 164)$ and boundary conditions

Figure 5: Computational mesh used for open turbine simulations.

model is seen to capture the pressure distribution more accurately than the fully turbulent assumption at these locations. At speeds beyond $U_{\infty}=10 \mathrm{~m} / \mathrm{s}$, the flow is deeply stalled over much of the turbine blade, leading to an inherently unsteady flow for which RANS based calculations are insufficient. Indeed, under such conditions, interaction between the blades and tower plays a significant role in the blade loading, and the tower surface must be included in calculations to produce reasonable results [30].

\section{Baseline (NACA0006) shroud}

An initial assessment of the shrouded turbine concept is performed by enclosing the NREL VI blade in a simple shroud. This shroud, referred to as "baseline," is generated by extruding a NACA0006 airfoil section at a $15^{\circ}$ angle of attack into an annular wing. The blade grid and background grid are the same as those used for validation. The dimensions for the shroud grid are $267 \times 201 \times 49$ in the wrap-around, spanwise, and normal directions respectively. Figure 7 (a) shows sections of the blade and shroud grids, and Figure 7(b) shows both the shroud and blade embedded in the background grid. Once again, periodic boundary conditions are used on the lateral faces of the domain, and characteristic farfield boundary conditions are used on the upstream, downstream, and farfield faces of the cylinder. A no-slip condition is applied on the blade in the rotating frame, and a separate no-slip condition enforcing zero velocity in the inertial frame is applied on the shroud surface. The clearance between the blade tip and shroud interior is $0.056 R$ or, expressed in the form common in turbomachinary applications, $0.785 c_{t}$ where $c_{t}$ is the chord length at the blade tip.

Results for the baseline shroud are shown in Figure 8. In this plot, error bars are used to represent the fluctuations (min and max) due to unsteady effects. Figure 8(a) plots the thrust coefficient for both the shrouded and open turbines. The coefficient is normalized in two ways: according to the turbine radius (solid line) and according to the maximum shroud radius (dashed line). In the presence of the shroud, the loads on the turbine are higher at all windspeeds. This is to be expected due to the acceleration induced by the shroud and should be considered when selecting materials for shrouded turbine blades. In place of power coefficient, a commonly used $[4,5]$ measure of DAWT performance is the augmentation ratio, given by

$$
r_{a}=\frac{C_{p}}{0.593}
$$

which is simply a ratio of the standard power coefficient to the Betz limit. The baseline shroud is seen to augment power significantly, with a maximum augmentation ratio of $r_{a}=1.09$ at $U_{\infty}=5 \mathrm{~m} / \mathrm{s}$. In this case, power extraction beyond the Betz limit is achieved even with a very simple shroud geometry. 


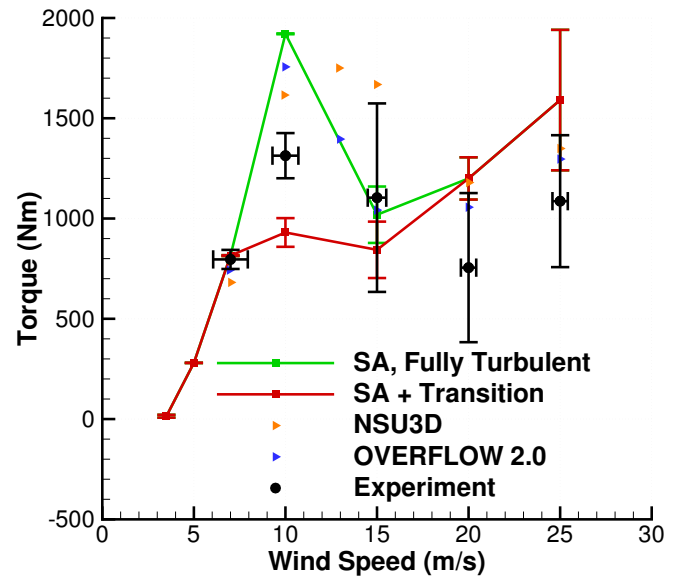

(a) Integrated torque vs. wind speed. OVERFLOW and NSU3D calculations from [28].

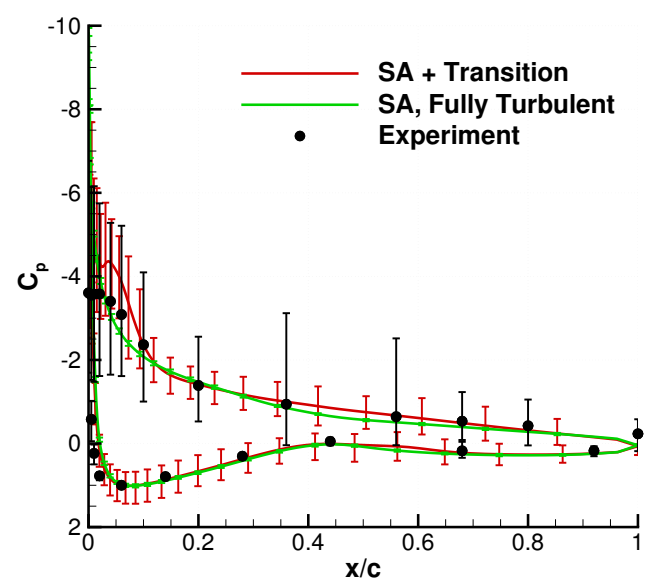

(c) $C_{p}$ distribution, $r / R=0.3, U_{\infty}=10 \mathrm{~m} / \mathrm{s}$

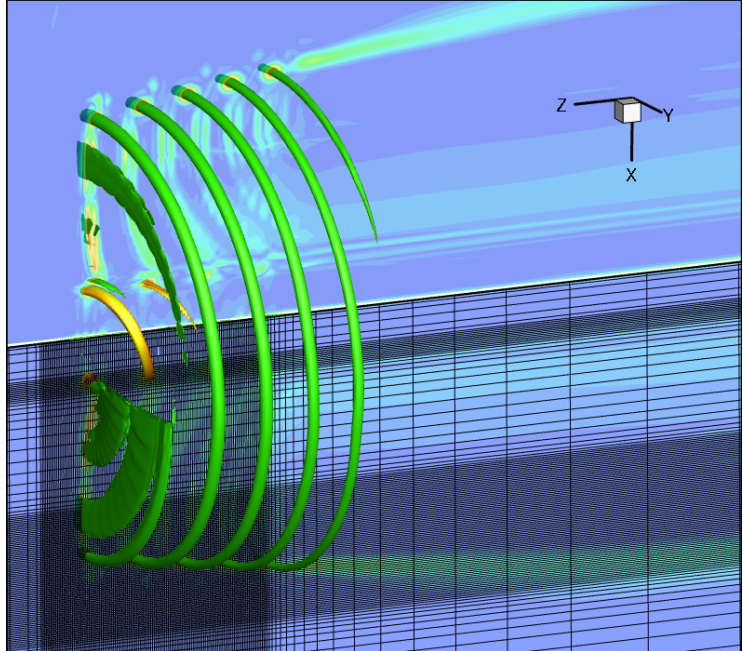

(b) Isocontour of q-criterion

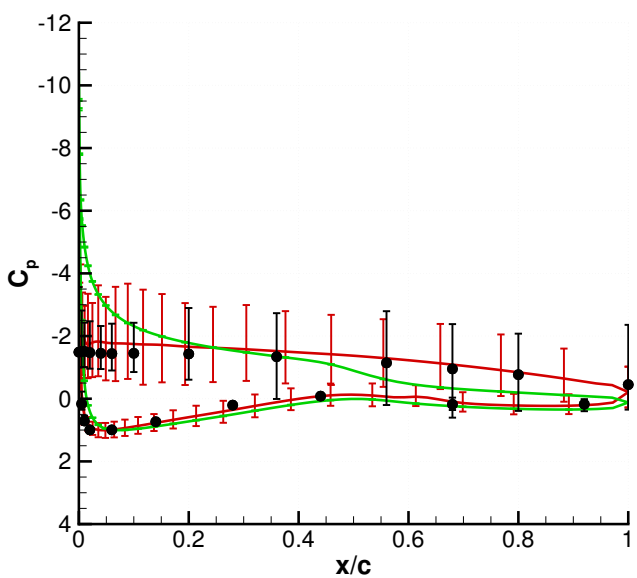

(d) $C_{p}$ distribution, $r / R=0.47, U_{\infty}=10 \mathrm{~m} / \mathrm{s}$

Figure 6: Validation of computational methodology including transition model on NREL Phase VI turbine. 


\section{2D Analysis of Shroud Airfoil Sections}

In the present work, only shrouds which are symmetric about an axis of rotation are considered. This permits the expedient assessment of shrouds generated by several different airfoil sections by solving the axisymmetric RANS equations. Following the work of previous authors [1,11], the effectiveness of a shroud is considered decoupled from the properties of a turbine, and the turbine is not modeled in this section. This conclusion is based on the assumptions of inviscid momentum theory, which will reach their useful limits when considering the interaction between a three-dimensional blade and shroud. Nonetheless, an axisymmetric investigation provides considerable insight into the properties of a shroud airfoil.

One measure of the effectiveness of an airfoil as a shroud profile is the amount of mass flow it induces through the interior of the shroud. For an axisymmetric shroud, an increased circulation would correspond to a large mass flow rate. The mass flow rate is given by

$$
\dot{m}=\int_{0}^{2 \pi} \int_{0}^{R_{s}} \rho u_{z} r d r d \theta
$$

where $u_{z}$ is the axial component of velocity. A non-dimensional mass flow amplification factor is defined as

$$
M_{r}=\frac{\dot{m}}{\rho U_{\infty} \pi R_{s}^{2}} .
$$

where the shroud radius $R_{s}$ is measured at the plane of the turbine. In the present work, for both $2 \mathrm{D}$ and $3 \mathrm{D}$ simulations the turbine was placed at $z / c=10 \%$, where $z$ is the axial coordinate from the leading edge and $c$ is the shroud chord length.

The Kutta-Joukowksi theorem states that the lift per span is proportional to the circulation induced in the surrounding flow. A high circulation corresponds directly to a high mass flow amplification. Considering this, several high-lift airfoils are considered. A sample of shapes considered are each shown alongside a NACA0006 profile in Figure 9(a). The Eppler E423 [31] is an airfoil designed to maximize lift through the use of a concave pressure recovery with no specific regard to the effect on moment coefficient. The modified NACA0006 was generated by rotating the mean camber line downward by $45^{\circ}$ for the last $20 \%$ of the chord length while maintaining the original thickness distribution. This shape is intended to resemble an airfoil with a deflected control surface, and it also approximates the flanged diffuser shape of Abe and Ohya [7, 10]. The Selig S1223 is designed with the same high-lift design philosophy as the Eppler E423 [31], and is intended to belong to the FX CL/MS-class of airfoils. The FX 74-CL4-140 is a high-lift airfoil designed for a Reynolds number of $R e=10^{6}$.

Two-dimensional overset grids are once again used for these computations, with $180 \times 218$ points in the background mesh and $267 \times 61$ points in the near-body mesh. A sample grid of an S1223 section at $10^{\circ}$

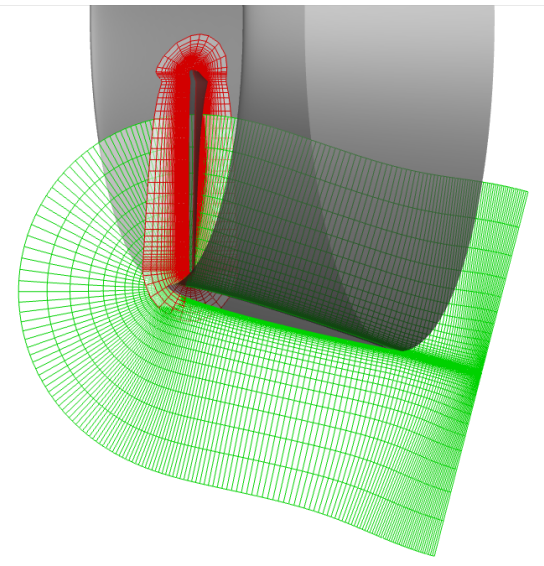

(a) View of blade $(257 \times 51 \times 51)$ and shroud $(267 \times 201 \times 49)$

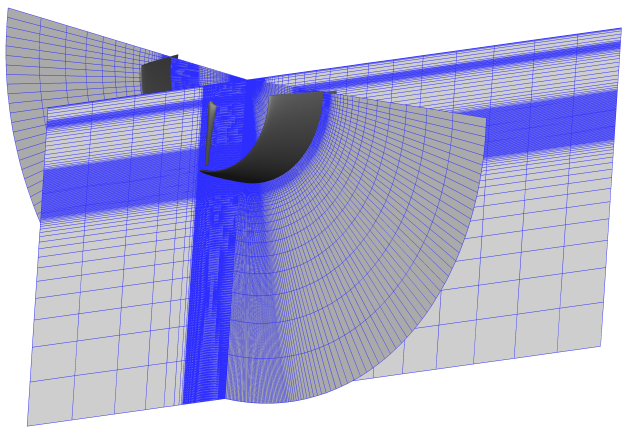

(b) Background grid $(201 \times 133 \times 164)$

Figure 7: Overset grids used for 3D shrouded turbine computation. 


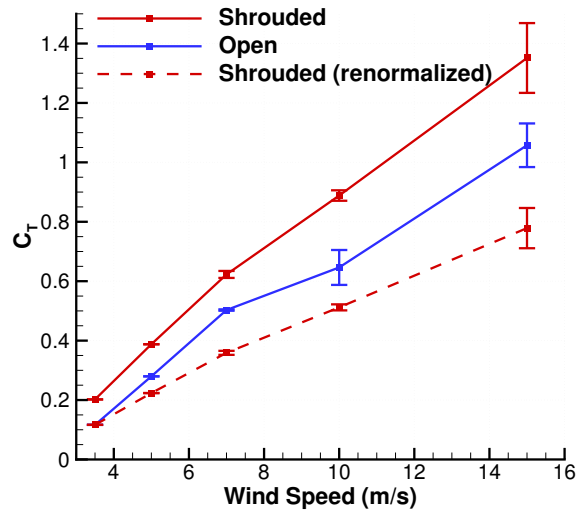

(a) Thrust coefficient

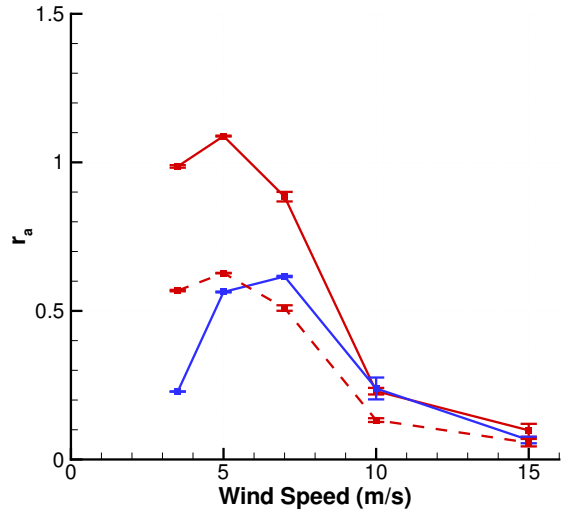

(b) Augmentation ratio

Figure 8: Turbine thrust and augmentation ratio with NACA0006 shroud ( $\omega$ fixed). Dashed lines are normalized with respect to maximum shroud radius.

angle of attack is shown in Figure 10. The Reynolds number based on shroud length is $R e=3.2 \times 10^{6}$, corresponding to a freestream velocity of $10 \mathrm{~m} / \mathrm{s}$ and shroud length of $5.029 \mathrm{~m}$. The axisymmetric RANS equations are solved along with the Spalart-Allmaras turbulence model. The background mesh is extended to 25 chord lengths upstream, 25 chord lengths laterally, and 50 chord lengths downstream. A small value of $r=0.001 \mathrm{~m}$ is used at the boundary near the symmetry axis, where an extrapolation boundary condition is applied.

The computed performance of these airfoils in terms of radial force and mass flow is shown in Figures 9(b) and $9(\mathrm{c})$. As suggested by the Kutta-Jakouwski theorem, the mass flow amplification increases nearly linearly with radial force coefficient. The angle of attack for this study is varied from $\alpha=0^{\circ}$ to $\alpha=15^{\circ}$, which is sufficient to observe the stall of each airfoil considered.

Two lines of the same slope are seen on the curves in Figure 9(b). The determining factor for which line a particular data point lies on is the location of the stagnation point. For instance, the lift coefficient for the NACA0006 section at $\alpha=6^{\circ}$ is nearly equal to that of the modified NACA0006 at $\alpha=0^{\circ}$, yet the NACA0006 induces a greater mass flow. This can be seen by examining the flowfields. Figures 11(a) and 11(b) show contours of pressure and streamlines near the leading edges of these two cases. In Figure 11(a), the stagnation point of the modified NACA0006 is located such that some of the flow approaching the leading edge of the airfoil is diverted downwards and around the shroud. By contrast, in Figure 11(b), the location of the stagnation point of the NACA0006 is such that approaching flow is pushed upwards and into the shroud, increasing the total mass flow. Similarly, Figures 11(c) and 11(d) show the leading edge of the S1223 airfoil at $\alpha=4^{\circ}$ and $\alpha=15^{\circ}$ respectively. Once again, the radial lift force is nearly equal for these two cases. However there is greater mass flow for $\alpha=15^{\circ}$ due to the location of the stagnation point.

Of the airfoils considered, the Selig S1223 achieves the greatest mass flow amplification, and for this reason it is selected for $3 \mathrm{D}$ analysis in the next section.

\section{High-lift shroud geometry}

Following the results of the previous section, a second three-dimensional shroud was generated with the cross section of the Selig S1223 airfoil. The grid dimensions and shroud radius were selected to be identical to those of the previous cases.

Computational results for the performance are shown in Figure 12 alongside the results obtained from the original NACA0006 geometry. The high-lift airfoil greatly improves the performance of the shrouded turbine, yielding a maximum augmentation ratio of $r=1.91$ at $U_{\infty}=5 \mathrm{~m} / \mathrm{s}$. This is an increase in performance of $75 \%$ over the baseline NACA0006 shroud at the windspeed of peak power extraction. The turbine still 


\section{Eppler E423}

Selig S1223

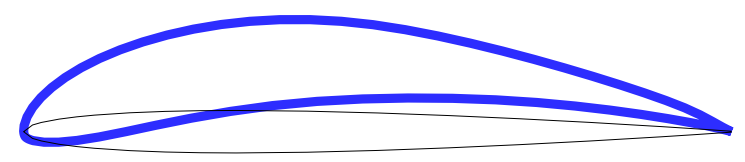

Modified NACA0006

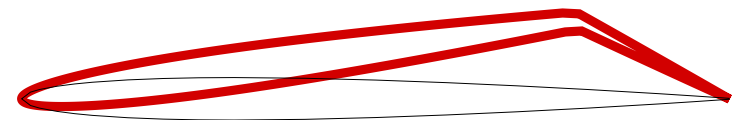

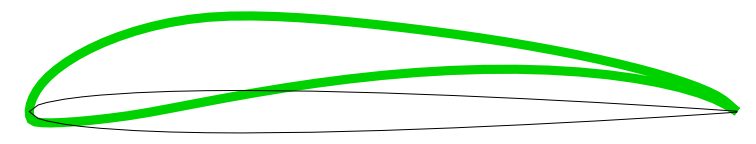

FX 74-CL5-140

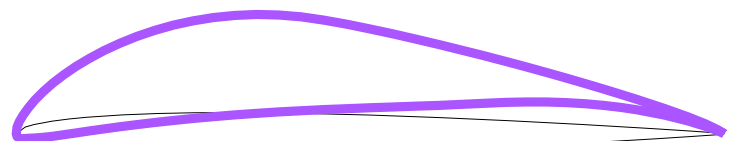

(a) Airfoil shapes

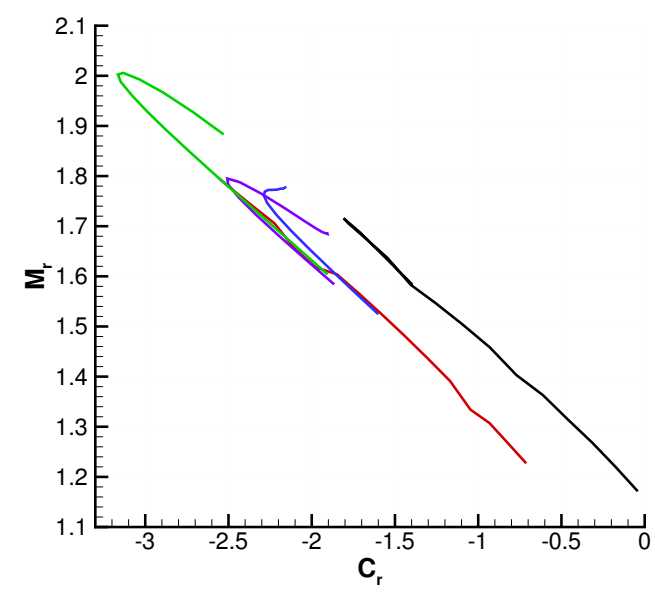

(b) Mass flow amplification $M_{r}$ vs. radial force coefficient $C_{r}$

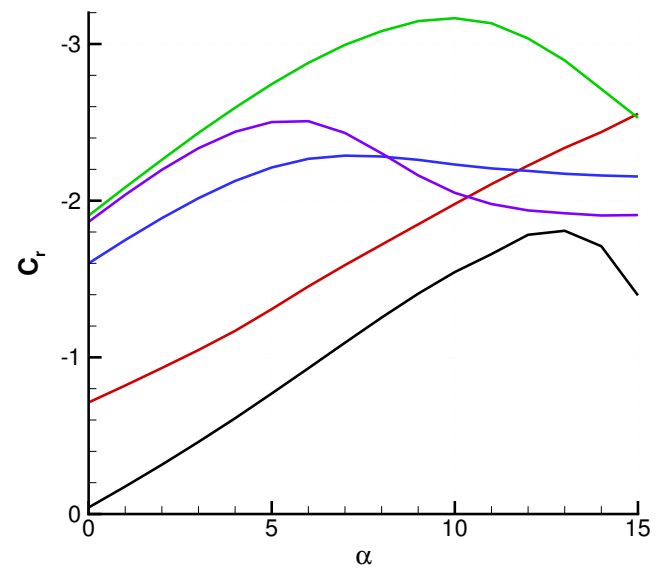

(c) Radial force coefficient $C_{r}$ vs. angle of attack $\alpha$

Figure 9: High-lift sections shapes and performance, black lines are NACA0006.

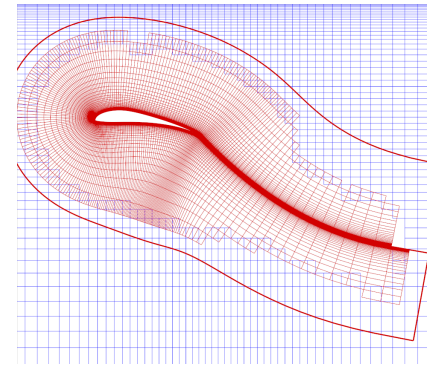

(a) Airfoil grid

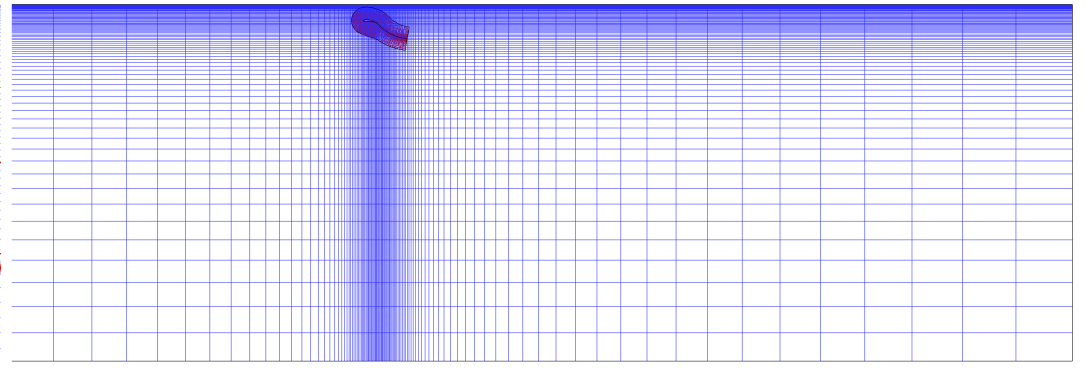

(b) Background grid

Figure 10: Overset grid system used for viscous axisymmetric computations.

achieves deep stall at $U_{\infty}=10 \mathrm{~m} / \mathrm{s}$, and once this high speed is reached the power augmentation of the shrouded turbines collapse to nearly the same value.

The effect of both shrouds on the spanwise distribution of loads on the turbine blade is shown in Figure 13. 


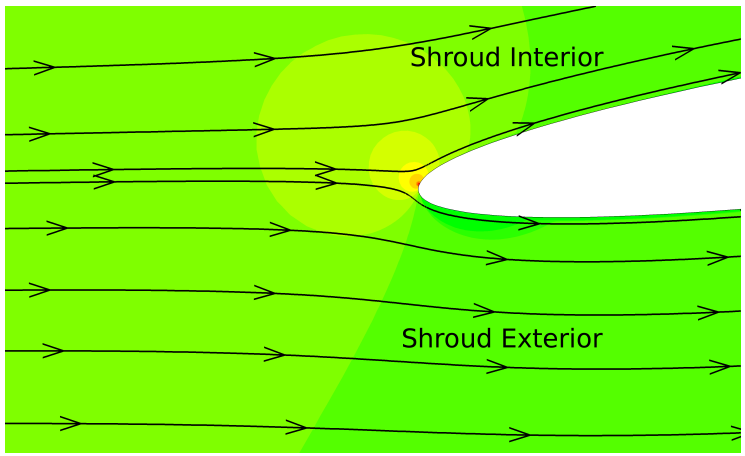

(a) Modified NACA0006 $\alpha=0^{\circ}$

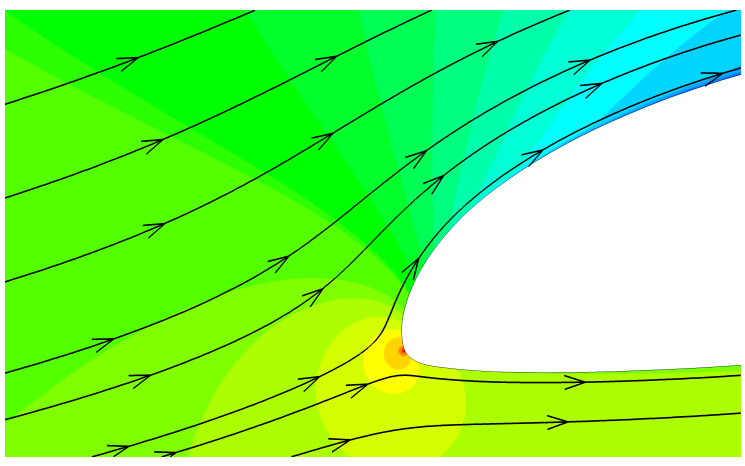

(c) $\mathrm{S} 1223 \alpha=4^{\circ}$

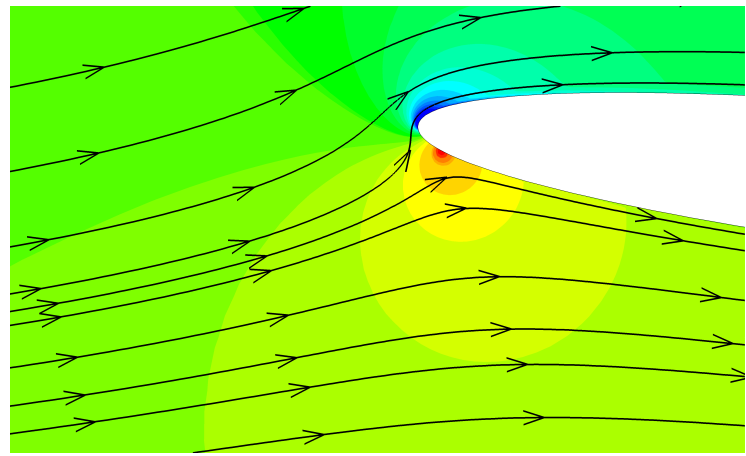

(b) NACA0006 $\alpha=6^{\circ}$

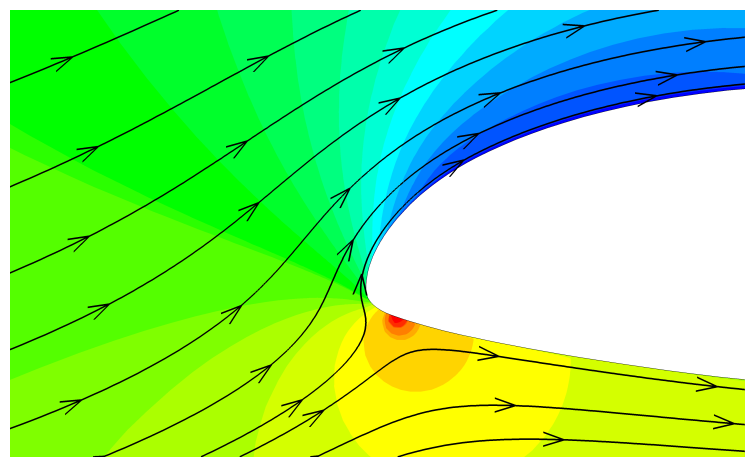

(d) $\mathrm{S} 1223 \alpha=15^{\circ}$

Figure 11: Stagnation point visualizations, contours of pressure.

Two wind speeds are shown, $U_{\infty}=5 \mathrm{~m} / \mathrm{s}$, where peak augmentation is obtained, and $U_{\infty}=7 \mathrm{~m} / \mathrm{s}$, which marks the onset of separation for the shrouded cases. At $U_{\infty}=5 \mathrm{~m} / \mathrm{s}$, where the turbine is more efficient in augmenting the power output, the increase in loading is smooth, peaking near $r / R=0.9$. At $U_{\infty}=7 \mathrm{~m} / \mathrm{s}$, the force coefficients which are smooth for the open rotor begin to deteriorate from the outboard portion of the blade. This is in part due to the fact that the result is unsteady.

To further investigate the irregularities seen in the outboard portion of the blade, Figure 14 shows contours of pressure and streamlines for a blade cross section taken at $r / R=0.7$ for $U_{\infty}=7 \mathrm{~m} / \mathrm{s}$. While the flow over the open turbine shown in Figure 14(a) remains fully attached, the flow over the shrouded turbine blade shown in Figure 14(b) exhibits flow separation. This explains the irregularities seen in Figure 13. It can also be seen in these plots that the local angle of attack is higher in the presence of the shrouded, indicating that the velocity normal to the blade surface, i.e. the windspeed, has increased.

\subsection{Flowfield}

In order to better understand the aerodynamics of a shrouded turbine, it is valuable to analyze the flowfield in some detail. First, the case with $U_{\infty}=5 \mathrm{~m} / \mathrm{s}$ is considered, as it corresponds to the windspeed of greatest power output.

Velocity profiles, from 2 rotor radii upstream to 1 radii downstream, are examined in Figure 15 for the open and shrouded rotor. These profiles have been averaged in the azimuthal direction. Upstream of the turbine, at $z / R=2$, the velocity profiles are constant at the freestream value of $5 \mathrm{~m} / \mathrm{s}$. At $z / R=0.5$, a deficit is seen in the wake of the open turbine corresponding to an expansion of incoming streamlines. At this same axial station, a peak is seen in the vicinity of the shroud (near $r / R=1$ ), and the flow acceleration due to the shroud prevents an upstream wake deficit. The incoming velocity is greater than the freestream velocity across the entire span of the turbine. At the plane of the turbine, at $z / R=0.0$, the wake of the open rotor has decelerated halfway to the downstream wake, agreeing with the result from actuator disk theory. The shroud, on the other hand, accelerates the freestream wind considerably. Downstream of the 


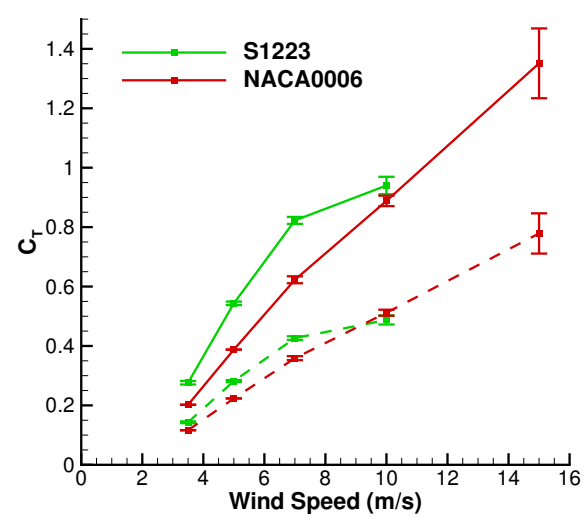

(a) Thrust

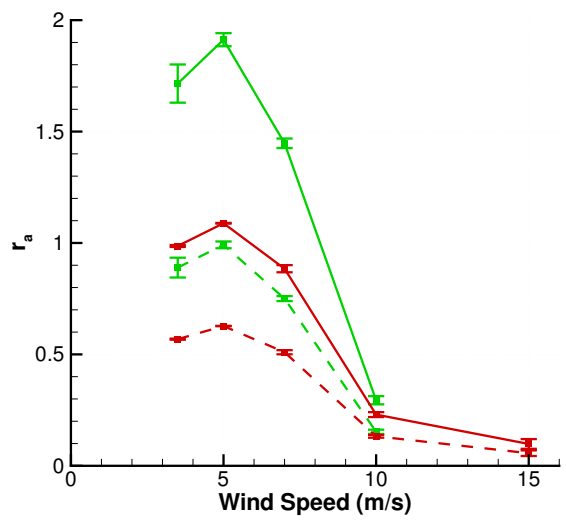

(b) Augmentation ratio

Figure 12: Thrust coefficient and augmentation ratio for NACA006 shroud and Selig S1223 shroud ( $\omega$ fixed). Dashed lines are normalized with respect to max shroud radius (as opposed to turbine radius).

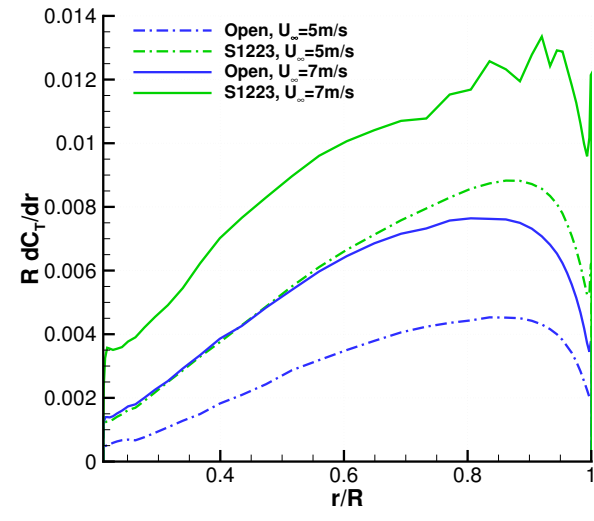

(a) Spanwise thrust distribution

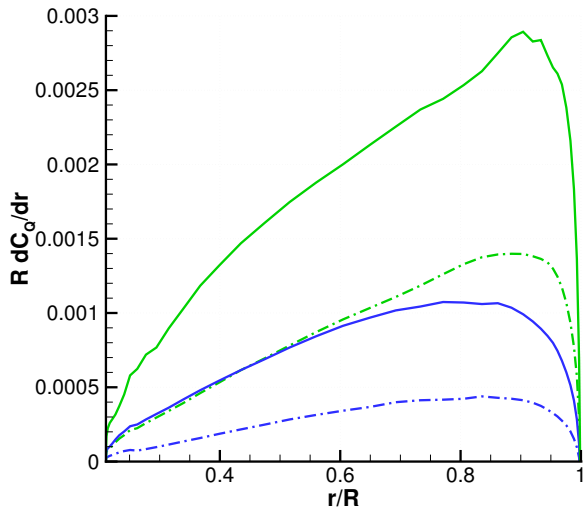

(b) Spanwise torque distribution

Figure 13: Spanwise thrust and torque distribution on open and shrouded blade. 


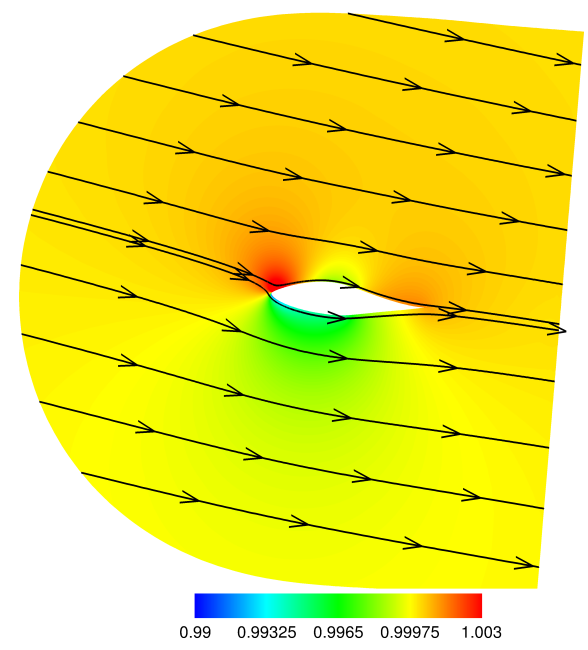

(a) Open turbine

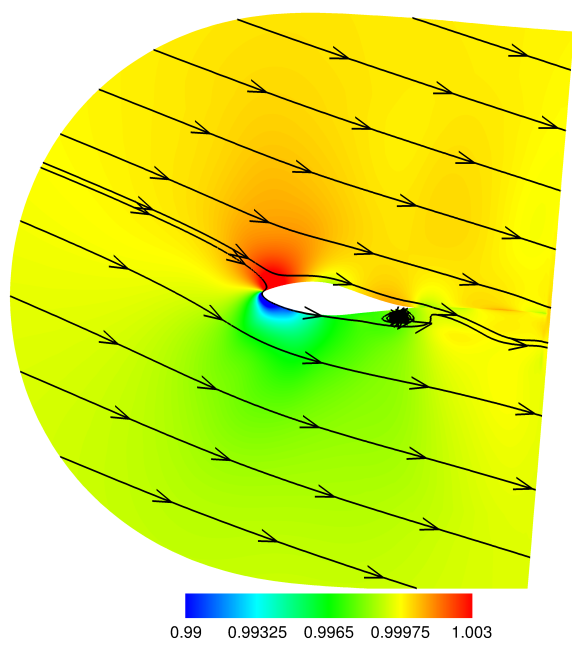

(b) Shrouded turbine (S1223)

Figure 14: Contours of $p / p_{\infty}$ at radial location $r / R=0.7, U_{\infty}=7 \mathrm{~m} / \mathrm{s}$.

turbine but ahead of the shroud trailing edge at $z / R=-0.5$, the wake of the open turbine has reduced to its downstream value, whereas the shroud contains and delays wake expansion. Farther downstream, the wake deficits are much larger for the shrouded turbine due to both an increase in turbine thrust and momentum extraction by the shroud itself. The shrouded turbine wake also expands farther laterally in this downstream region, indicating a greater diffusion of momentum, which would reduce the wake effect felt by a downstream turbine in a wind farm. The NREL Phase VI blade under consideration in the present work is designed to operate in a nearly uniform velocity distribution like that seen in Figure 15(a). The large deviation from a flat profile seen in Figure 15(b) indicates that a redesign of the blade is needed for shrouded turbines. Such a redesign is deferred to future work.

Contours of the normalized pressure and skin friction along the turbine blades are shown in Figure 16 for both the open and shrouded cases. The differences between the cases are most pronounced on the leeward surface. In the plots on the left, lower pressures are seen towards the outer region of the blade in the shrouded case, where the effect of the shroud suction peak is felt most strongly. Streamlines indicating the flow direction immediately adjacent to the surface are superimposed on these plots. Laminar separation bubbles, predicted by the transition model, are seen as blue stripes across the blades. At these locations, the skin friction is negative and locally the flow direction is reversed. Substantial cross flow and some leading edge separation are present in the shrouded case.

Interesting features are seen in the interaction between the turbine wake and the shroud. Figure 17(a) shows contours of vorticity magnitude taken at an azimuthal cross section in the vicinity of the blade for $U_{\infty}=5 \mathrm{~m} / \mathrm{s}$. Downstream of the turbine, the passing helical turbine wake appears in close proximity to the shroud, revealing the expansion of the helix within the shroud. The vorticity shed from the shroud boundary layer oscillates with the passing wake. The fluid in the boundary layer decelerates toward pressure recovery but is intermittently reenergized by the passing turbine vortex. This phenomenon promotes attached flow, and is quite beneficial in preventing stall on the shroud inner surface. Figure 17(b) shows a simlar plot for $U_{\infty}=7 \mathrm{~m} / \mathrm{s}$. In this case, there is substantial flow separation along the turbine blade, and a large and irregular wake is shed into the shroud.

The pressure coefficient along the shroud is plotted in Figure 18 for wind speeds of $U_{\infty}=5 \mathrm{~m} / \mathrm{s}$ and $U_{\infty}=$ $7 \mathrm{~m} / \mathrm{s}$. Pressure coefficient distributions at various azimuthal locations are displayed alongside the azimuthally averaged distribution. The solution of a $2 \mathrm{D}$ axisymmetric simulation is shown as well for comparison. As depicted in Figure 18(c), the location where the azimuth $\theta=0^{\circ}$ corresponds to the location of the turbine blade. In both cases, a large peak in suction is seen at this location, as the suction peak of the shroud interacts with the tip vortex shed by the turbine. The effect of the passing helical wake is readily visible as oscillations in these plots. 


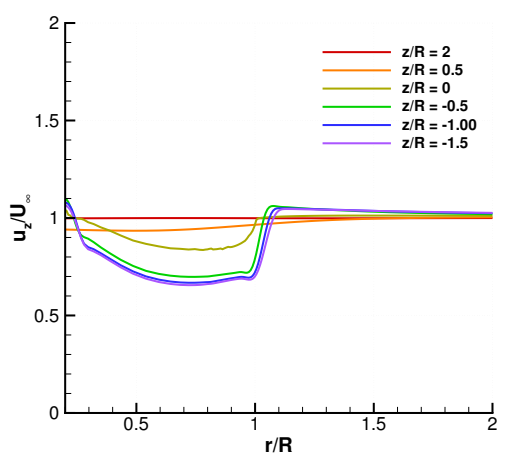

(a) Open turbine

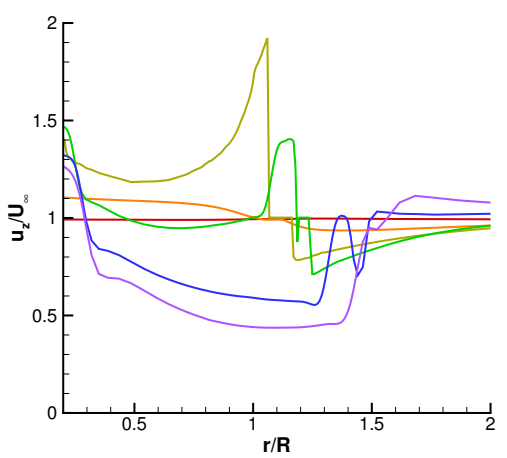

(b) S1223 shroud

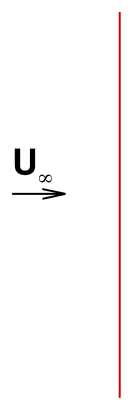

Z/R: 2

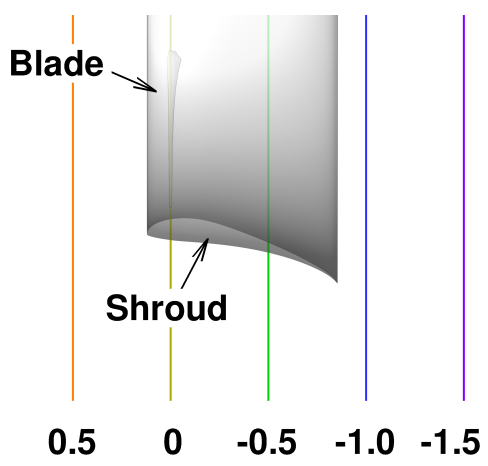

(c) Locations of axial stations for Figures 15(a) and 15(b)

Figure 15: Azimuthally averaged velocity distribution, $U_{\infty}=5 \mathrm{~m} / \mathrm{s}$.

\subsection{Varying tip speed ratio at a fixed wind speed}

In practice, it is typically desirable to select a rotor rotation rate $\omega$ to match the rate at which the gearbox and power generator perform optimally. Nonetheless, rotation rate plays a crucial role in the aerodynamics of a wind turbine system. In Figure 8(b), the power produced by the shrouded turbine while maintaining a fixed $\omega$ decreases with increasing windspeed beginning at $U_{\infty}=7 \mathrm{~m} / \mathrm{s}$. At and beyond this speed, there is flow separation on the blade surface owing to the higher angle of attack experienced by each blade section. The geometric angle of attack is given by

$$
\alpha(r)=\phi(r)+\tan ^{-1}\left(\frac{1}{\lambda} \frac{R}{r}\right)
$$

where $\phi(r)$ is the twist of the blade. Inspection of this relation confirms that the local angle of attack can be reduced all along the blade by increasing $\lambda$. In other words, it is possible to reduce the extent of separation on a blade and reduce it altogether by increasing the rotation rate.

Performance results from computations run at a fixed speed of $U_{\infty}=7 \mathrm{~m} / \mathrm{s}$ at various tip speed ratios are shown in Figure 19. The lowest value of $\lambda=5.42$ corresponds to the original conditions of the NREL Phase VI, and successive runs increase this value by $25 \%$ each. Figure 19(b) reveals that there is an optimum value of $\lambda$ for power production.

An examination of the flowfields reveals that three physical phenenomenon play a role in the power output of this system:

- The presence and extent of flow separation on the turbine blade

- Profile drag of the blade 
Windward Surface

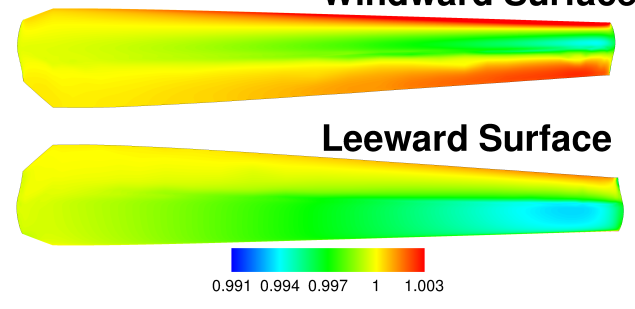

(a) Open turbine, contours of $p / p_{\infty}$

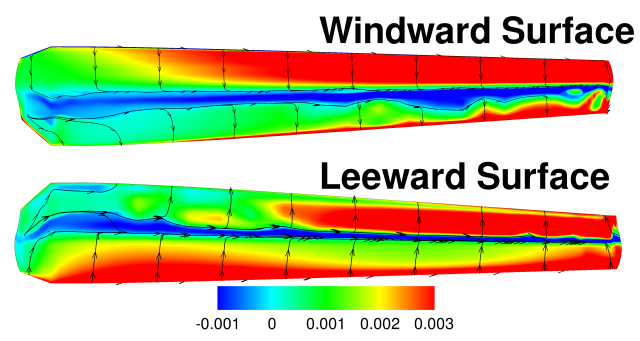

(b) Open turbine, contours of $c_{f}$

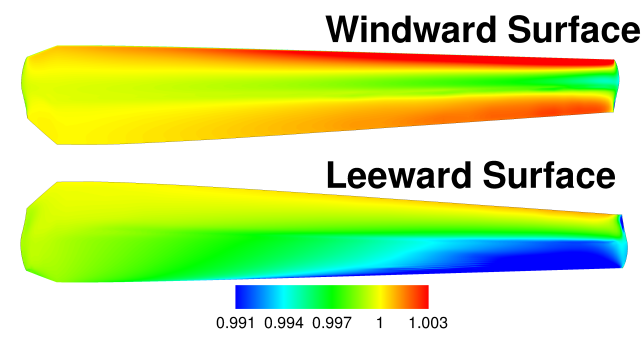

(c) S1223 shroud, contours of $p / p_{\infty}$

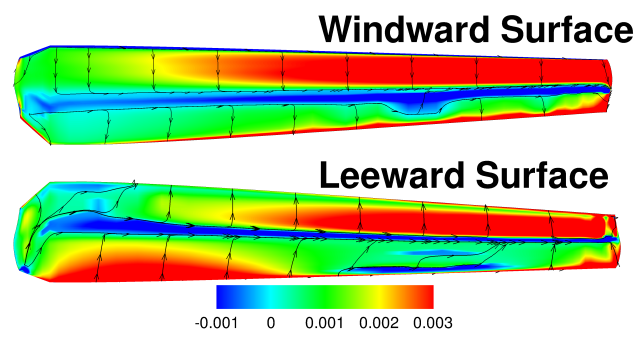

(d) S1223 shroud, contours of $c_{f}$

Figure 16: Surface pressure and skin friction contours, $U_{\infty}=5 \mathrm{~m} / \mathrm{s}$.

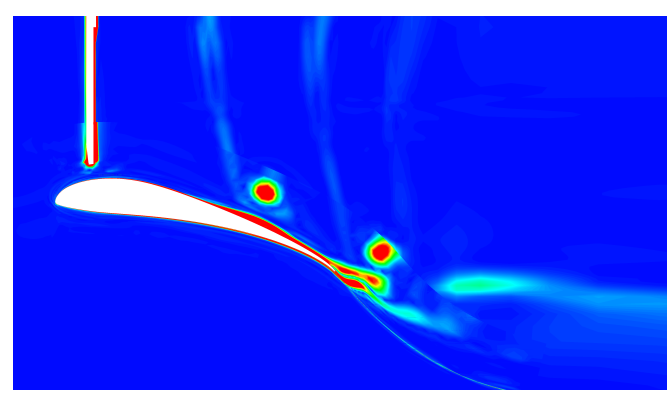

(a) $U_{\infty}=5 \mathrm{~m} / \mathrm{s}$

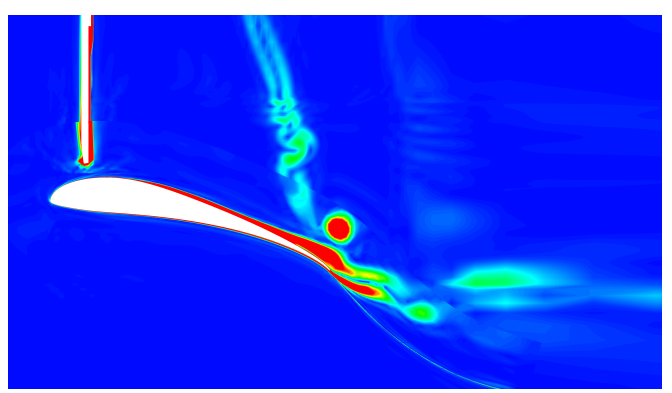

(b) $U_{\infty}=7 \mathrm{~m} / \mathrm{s}$

Figure 17: Contours of vorticity magnitude for S1223 shroud. 


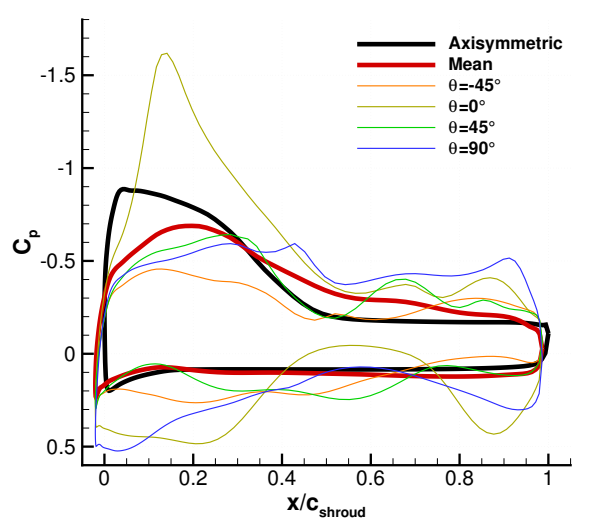

(a) $U_{\infty}=5 \mathrm{~m} / \mathrm{s}$

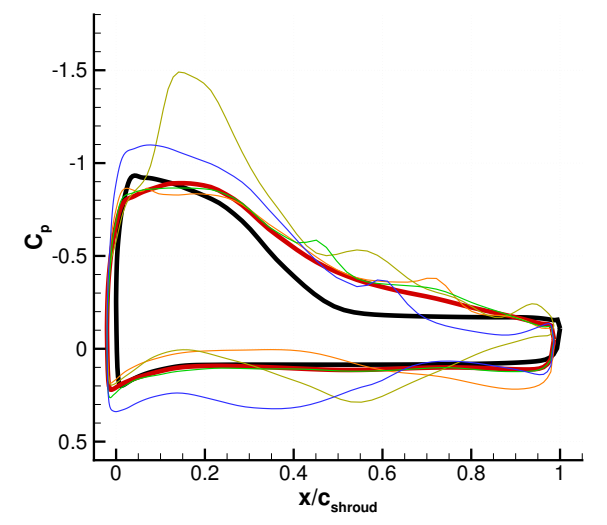

(b) $U_{\infty}=7 \mathrm{~m} / \mathrm{s}$

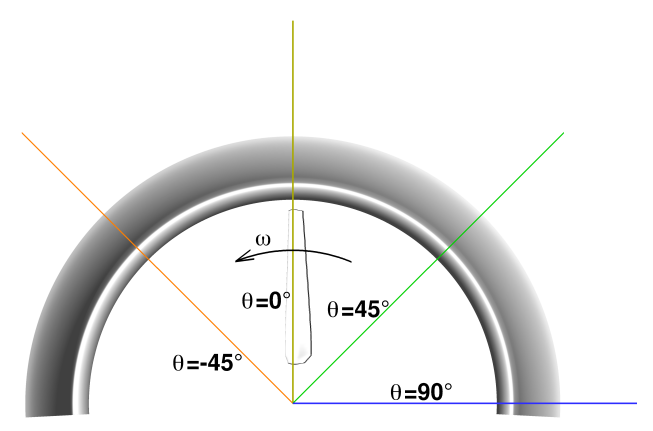

(c) Azimuthal stations

Figure 18: Pressure coefficient along shroud. Mean value shown alongside several stations. Axisymmetric solution shown for comparison. 


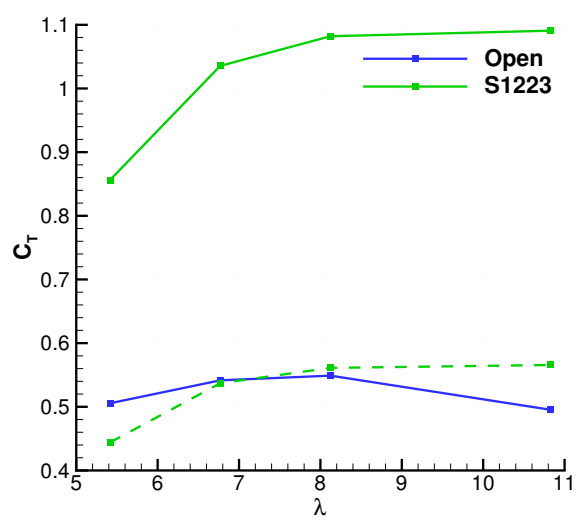

(a) Thrust coefficient

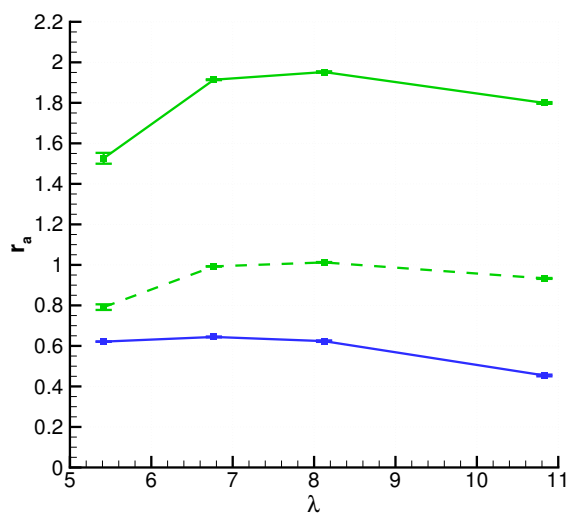

(b) Augmentation ratio

Figure 19: Thrust coefficient and power augmentation for varying tip speed ratios at a fixed freestream velocity of $U_{\infty}=7 \mathrm{~m} / \mathrm{s}$.

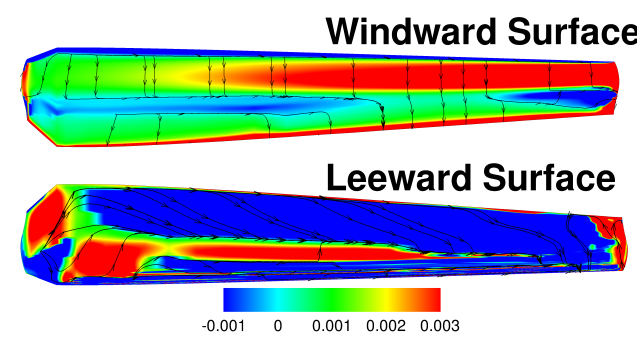

(a) $\lambda=5.42$

Windward Surface

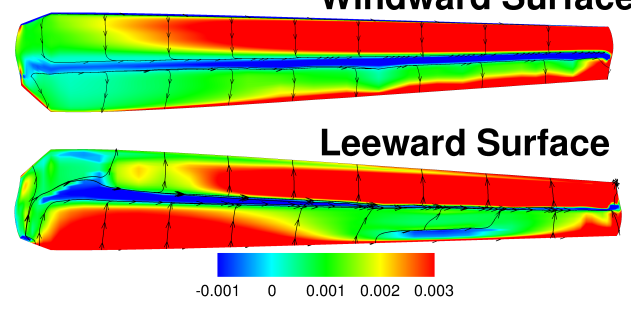

(c) $\lambda=8.12$

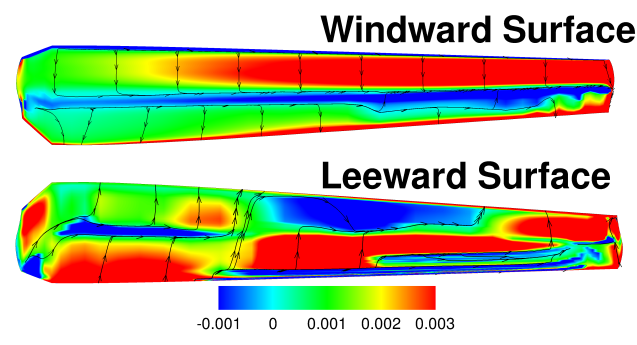

(b) $\lambda=6.77$

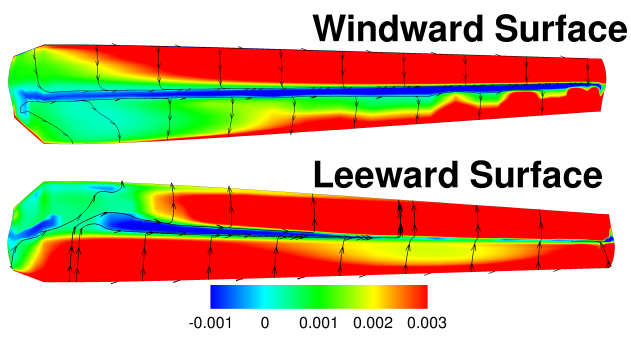

(d) $\lambda=10.8$

Figure 20: Contours of skin friction for varying tip speed ratios, S1223 shroud.

- The performance of the shroud in the wake of the turbine

The first of these items causes performance to improve as $\lambda$ increases while the latter two cause performance to decrease with increasing $\lambda$.

Considering first the extent of flow separation, Figure 20 shows skin friction at the blade surface along with surface streamlines. At the baseline tip speed ratio, shown in Figure 20(a), the flow past the leeward surface is highly separated and much of it is reversed. By increasing $\lambda$ by 25\%, shown in Figure 20(b), the extent of separation is greatly reduced although there are still large separation regions on the leeward surface near the leading and trailing edges. By increasing $\lambda$ further, in Figures 20(c) and 20(d), separation is eliminated except for the thin laminar separation bubbles cutting across the span of the blades.

Next, the profile drag on a shrouded turbine increases with increasing tip speed ratio. Neglecting three- 


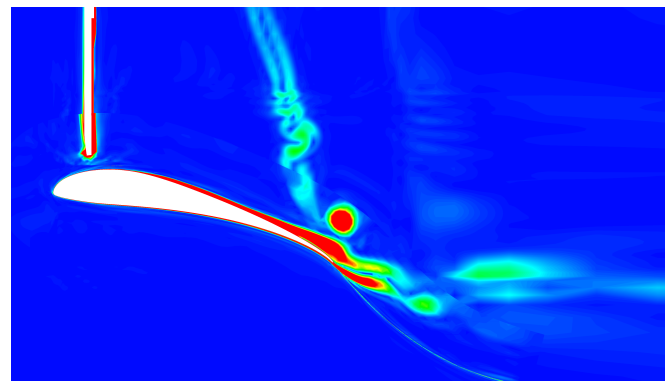

(a) $\lambda=5.42$

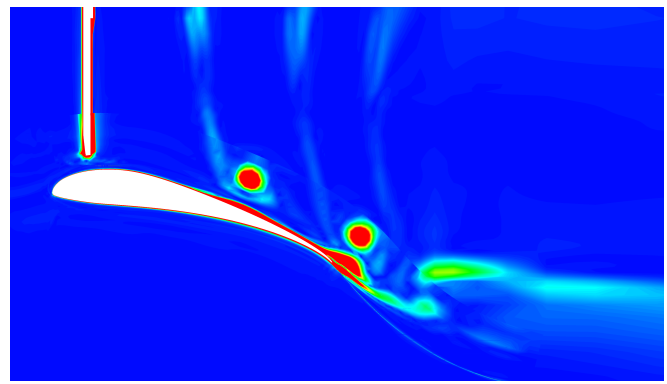

(c) $\lambda=8.12$

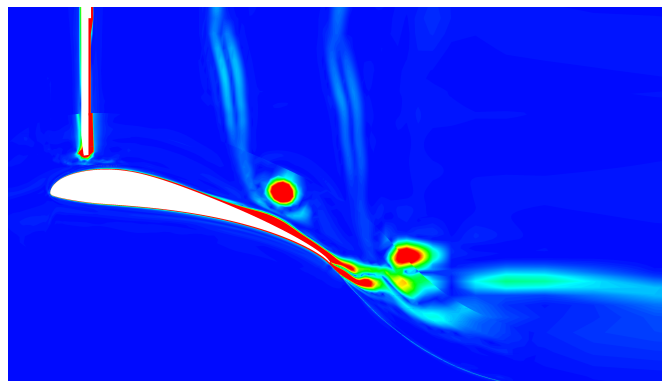

(b) $\lambda=6.77$

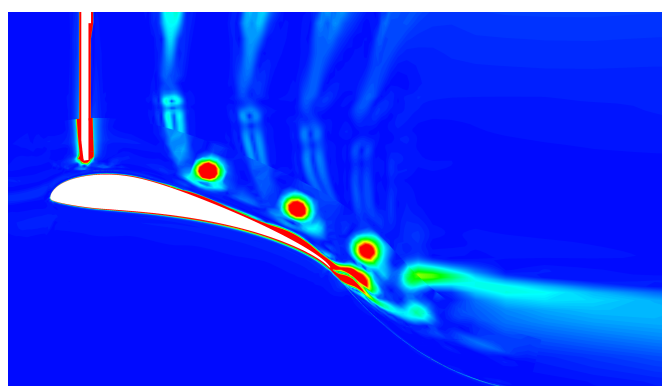

(d) $\lambda=10.8$

Figure 21: Contours of vorticity magnitude for varying tip speed ratios, S1223 shroud.

dimensional effects, the drag on a section of a turbine blade is given by

$$
d=C_{d} \frac{1}{2} \rho\left[(\omega r)^{2}+U_{\infty}^{2}\right]=C_{d} \frac{1}{2} \rho U_{\infty}^{2}\left[\left(\frac{\lambda r}{R}\right)^{2}+1\right] .
$$

Thus, profile drag is proportional to $\lambda^{2}$ and can be expected to grow significantly as $\lambda$ increases.

Finally, the mass flow amplification of the shroud is also affected by an increase in $\lambda$. As the tip speed ratio of a wind turbine increases, the distances between successive passes of the tip vortex in the wake decreases. For a shrouded turbine, this means that more turns of the wake are present in the shroud interior. This can be seen in Figure 21, which plots the magnitude of vorticity near the shroud surface in the vicinity of the turbine blade. Increasing the tip speed ratio draws wake vortices closer to the leading edge of the shroud airfoil and increases the number of times the wake interacts with the shroud boundary layer.

The impact of the increased interaction between the turbine wake and the shroud is examined in Figure 22, which shows the pressure distribution along the shroud for each tip speed ratio considered. (The location of the azimuthal stations in these plots is shown in Figure 18(c)). The area inside the curve representing the mean $C_{p}$ distribution measures the lift force on the shroud and is an indicator of shroud performance. This area decreases with increasing $\lambda$, indicating that the impact of the wake is to reduce the performance of the shroud as a flow accelerator. Indeed, examination of velocity profiles (not shown) for these cases reveals that the amount of acceleration achieved by the shroud at the turbine plane decreases with increasing $\lambda$.

At the highest tip speed ratio considered, in Figure 22(c), an especially intense suction peak appears in the vicinity of the blade $\left(\theta=0^{\circ}\right)$. However, the mean suction is lower for this case than any other. This signifies the presence of strong pressure gradients in the azimuthal direction in this case, and the impact of this high suction peak is to set the flow swirling rather than draw flow axially through the shroud.

Of the three phenemena discussed above, the extent of flow separation outweighs the other two in importance up to the point where the flow is fully attached on the blade. Beyond this point, increasing $\lambda$ further only increases the profile drag and reduces the shroud efficiency. 


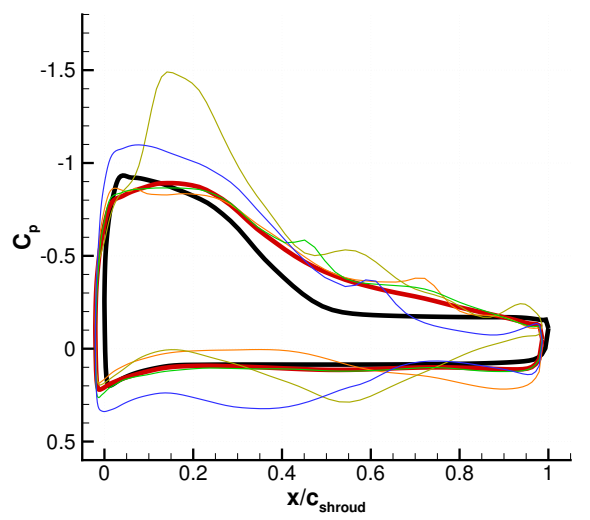

(a) $\lambda=5.42$

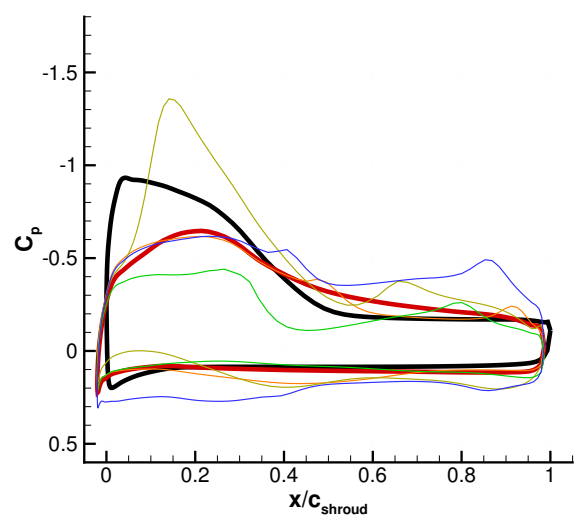

(c) $\lambda=8.12$

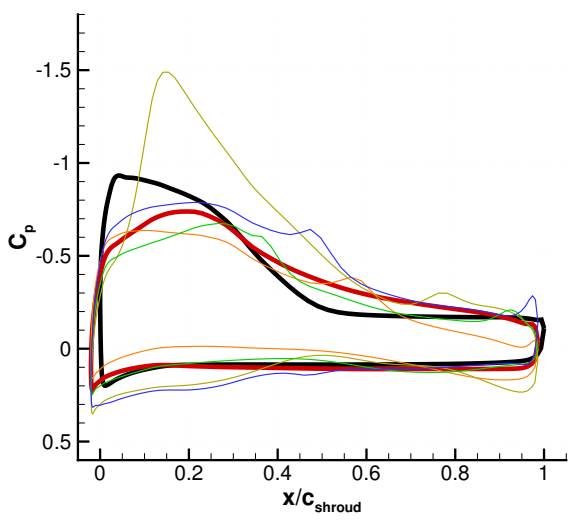

(b) $\lambda=6.77$

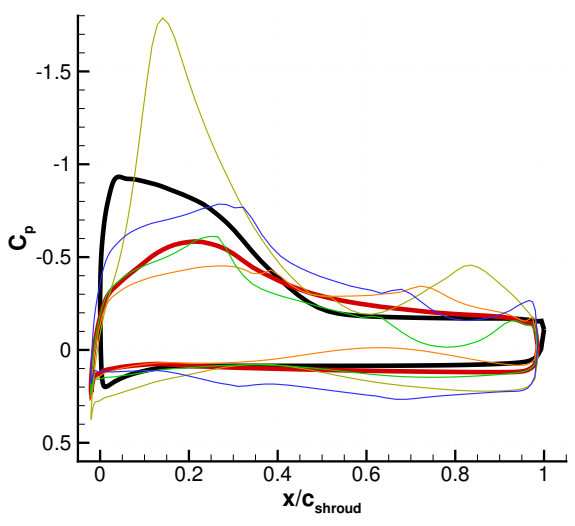

(d) $\lambda=10.8$

Figure 22: Pressure coefficient along shroud. Mean value shown alongside several stations. Axisymmetric solution shown for comparison. 


\section{Conclusions and future work}

The performance and flowfield of a shrouded wind turbine has been computed and analyzed in detail. High-lift airfoil shapes are considered for shroud geometry. Validation has been performed for the RANS based model used for computations, and the use of a transition model is found to improve the accuracy of results.

An investigation of airfoil sections for shrouds of shrouded wind turbines has been performed. Of a number of airfoil shapes considered for shroud cross sectional profiles, the Selig S1223 attains the greatest amplification of mass flow. Three-dimensional analysis of full shrouded turbine systems verify the benefit of increased mass flow through the plane of the turbine. Power extraction up to $90 \%$ beyond the Betz limit is achieved. The improvement in power extraction beyond the bare turbine is substantial; the NACA0006 shroud improves power over the bare turbine by a factor of 1.93 and the S1223 improves it by a factor of 3.39 at $U_{\infty}=5 \mathrm{~m} / \mathrm{s}$. These results further support the utility of the shrouded wind turbine as a device that can be used effectively at low cut-in speeds and offer promise to substantially improve the energy capture when compared to conventional wind turbines.

Based on the preceding analysis, the following conclusions are drawn regarding the design of a shrouded turbine:

- In the absence of a turbine, mass flow amplification through a shroud increases approximately linearly with radial force, and nonlinear behavior occurs as the stagnation point moves from the interior to the exterior side of the shroud.

- The wake of a shrouded turbine expands more rapidly than an open turbine and can be tailored to promote maximum power extraction.

- Transition has an important effect on the behavior of a shrouded wind turbine and should be accounted for in analysis whenever feasible.

- The shrouded turbine system's performance is maximum at a particular tip speed ratio. Increasing the tip speed ratio beyond this optimal value leads to losses due to separation.

The above considerations provide insight for both the analysis and design of both the shroud and the turbine of a shrouded turbine. Future work will employ an optimization strategy to design a shrouded turbine with a continued detailed focus on fluid physics. This procedure will include a redesign of the twist, chord, and thickness distribution of the blade. Additionally, the results presented here shall be compared to lowerorder theories, such as the commonly-used actuator disk model. It will also be interesting to consider the effect of extreme loads on such a system, such as a high-speed gust at a high angle of attack.

\section{Acknowledgement}

This work is supported by the DoD NDSEG fellowship and by the DoE ASCR program on Fluid/Structure interactions in Wind Turbine Applications at Stanford University.

[1] Jamieson, P., "Beating Betz: Energy Extraction Limits in a Constrained Flow Field," Journal of Solar Energy Engineering, Vol. 131, 2009.

[2] van Bussel, G., "The science of making more torque from wind: Diffuser experiments and theory revisited," Journal of Physics: Conference Series, Vol. 75, 2007.

[3] Lilley, G. M. and Rainbird, W. J., "A preliminary Report on the Design and Performance of a Ducted Windmill, Report 102," College of Aeronautics, 1956.

[4] Gilbert, B. L. and Foreman, K. M., "Experiments With a Diffuser-Augmented Model Wind Turbine," Journal of Energy Resources Technology, Vol. 105, 1983.

[5] Igra, O., "Research and Development for Shrouded Wind Turbines," Energy cons. 83 Mgmt, Vol. 21, 1981, pp. 13-48. 
[6] Hansen, M. O. L., Sørensen, N. N., and Flay, R. G. J., "Effect of Placing a Diffuser Around a Wind Turbine," Wind Energy, Vol. 3, 2000.

[7] Abe, K. and Ohya, Y., "An investigation of flow fields around flanged diffusers using CFD," Journal of Wind Engineering and Industrial Aerodynamics, Vol. 92, 2004.

[8] Abe, K., Nishida, M., Sakurai, A., Ohya, Y., Kihara, H., Wada, E., and Sato, K., "Experimental and numerical investigations of flow fields behind a small wind turbine with a flanged diffuser," Journal of wind engineering and industrial aerodynamics, Vol. 93, No. 12, 2005, pp. 951-970.

[9] Ohya, Y., Karasudani, T., Sakurai, A., Abe, K.-i., and Inoue, M., "Development of a shrouded wind turbine with a flanged diffuser," Journal of wind engineering and industrial aerodynamics, Vol. 96, No. 5, 2008, pp. 524-539.

[10] Ohya, Y. and Karasudani, T., "A Shrouded Wind Turbine Generating High Output Power with Windlens Technology," Energies, Vol. 131, 2010.

[11] Werle, M. J. and Presz Jr., W. M., "Ducted Wind/Water Turbines and Propellers Revisited," Journal of Propulsion and Power, Vol. 24, No. 5, 2008.

[12] Baeder, J., Duraisamy, K., and Lakshminarayan, V., "RANS Predictions of Complex Hovering Rotor Configurations: From Micro Scale to Full Scale," Computational Fluid Dynamics Journal, Vol. 18, No. $3-4,2011$.

[13] Duraisamy, K., Ramasamy, M., Baeder, J., and Leishman, G., "High Resolution Wake Capturing Methodology for Hovering Rotor Simulations," Journal of the American Helicopter Society, Vol. 52, No. 2, 2007.

[14] Duraisamy, K., Ramasamy, M., Baeder, J., and Leishman, G., "High Resolution Computational and Experimental Study of Hovering Rotor Tip Vortex Formation," AIAA Journal, Vol. 45, No. 11, 2007.

[15] Lakshminarayan, V. K., "Computational Investigation of Micro-Scale Coaxial Rotor Aerodynamics in Hover," Ph.D. dissertation, Department of Aerospace Engineering, 2009.

[16] Lakshminarayan, V. K. and Baeder, J. D., "Computational Investigation of Microscale Shrouded Rotor Aerodynamics in Hover," Journal of the American Helicopter Society, 2011, 56, 042002.

[17] Aranake, A., Lakshminarayan, V., and Duraisamy, K., "Assessment of Transition Model and CFD Methodology for Wind Turbine Flows," $42^{\text {nd }}$ AIAA Fluid Dynamics Conference and Exhibit, New Orleans, LA, June 2012.

[18] Medida, S. and Baeder, J., "Numerical Prediction of Static and Dynamic Stall Phenomena using the $\gamma-\overline{R e_{\theta t}}$ Transition Model," American Helicopter Society $67^{\text {th }}$ Annual Forum, Virginia Beach, VA, May 2011.

[19] Aranake, A., Lakshminarayan, V., and Duraisamy, K., "Computational Analysis of Shrouded Wind Turbine Configurations," 51st AIAA Aerospace Sciences Meeting, Dallas, TX, January 2013.

[20] Buelow, P. E. O., Schwer, D. A., Feng, J., and Merkle, C. L., "A Preconditioned Dual-Time, Diagonalized ADI scheme for Unsteady Computations," 13th AIAA Computational Fluid Dynamics Conference, Snowmass Village, CO, June 1997.

[21] Pandya, S. A., Venkateswaran, S., and Pulliam, T. H., "Implementation of Preconditioned Dual-Time Procedures in OVERFLOW," 41st AIAA Aerospace Sciences Meeting and Exhibit, Reno, NV, January 2003.

[22] Pulliam, T. and Chaussee, D., "A Diagonal Form of an Implicit Approximate Factorization Algorithm," Journal of Computational Physics, Vol. 39, No. 2, 1981, pp. 347-363.

[23] Turkel, E., "Preconditioning Techniques in Computational Fluid Dynamics," Annual Review of Fluid Mechanics, Vol. 31, 1999, pp. 385-416. 
[24] Spalart, P. R. and Allmaras, S. R., "A One-equation Turbulence Model for Aerodynamic Flows," 30th AIAA Aerospace Sciences Meeting and Exhibit, Reno, NV, January 1992.

[25] Lee, Y., "On Overset Grids Connectivity and Vortex Tracking in Rotorcraft CFD," Ph.D. dissertation, Department of Aerospace Engineering, 2008.

[26] Simms, D., Schreck, S., Hand, M., and Fingersh, L. J., "NREL Unsteady Aerodynamics Experiment in the NASA-Ames Wind Tunnel: A Comparison of Predictions to Measurements," NREL/TP-500-29494, 2001.

[27] Somers, D. M., "Design and Experimental Results for the S809 Airfoil," NREL/SR-440-6918, 1997.

[28] Potsdam, M. A. and Mavriplis, D. J., "Unstructured mesh CFD aerodynamic analysis of the NREL Phase VI rotor," 47th AIAA Aerospace Sciences Meeting, 2009.

[29] Jeong, J. and Hussain, F., "On the identification of a vortex," Journal of Fluid Mechanics, Vol. 285, No. 69, 1995, pp. 69-94.

[30] Zahle, F., Sørensen, N. N., and Johansen, J., "Wind turbine rotor-tower interaction using an incompressible overset grid method," Wind Energy, Vol. 12, No. 6, 2009, pp. 594-619.

[31] Selig, M. S. and Guglielmo, J. J., "High-Lift Low Reynolds Number Airfoil Design," Journal of Aircraft, Vol. 34, No. 1, 1997. 